\title{
Long non-coding RNA BCYRN1 promotes prostate cancer progression via elevation of HDAC11
}

\author{
WEI HUO $^{1 *}$, FEI QI $^{2 *}$ and KAICHEN WANG ${ }^{1}$ \\ Departments of ${ }^{1}$ Urology and ${ }^{2}$ Surgery, China-Japan Union Hospital, Jilin University, Changchun, Jilin 130033, P.R. China
}

Received November 18, 2019; Accepted March 12, 2020

DOI: $10.3892 / o r .2020 .7680$

\begin{abstract}
Prostate cancer is a serious threat to men's health worldwide. While previous studies have demonstrated that long non-coding RNAs (lncRNAs) are closely associated with the initiation and development of several types of cancer, the role of lncRNAs in the progression of prostate cancer remains incompletely understood. In the present study, the lncRNA brain cytoplasmic RNA 1 (BCYRN1) was found to be overexpressed in prostate tumors compared with healthy tissues. Furthermore, the expression of BCYRN1 was found to be associated with Gleason score and lymph node metastasis. It was demonstrated that BCYRN1 silencing using small interfering RNA (siRNA) inhibited the proliferation of prostate cancer cells. The results of the present study indicated the presence of a reciprocal regulatory association between BCYRN1 and microRNA (miR)-939-3p. In addition, it was observed that BCYRN1 directly sponged miR-939-3p to upregulate histone deacetylase 11 (HDAC11) expression in prostate cancer cells. Moreover, transfection of recombinant HDAC11 reversed the inhibition of cell proliferation that was induced by BCYRN1 siRNA. A positive correlation between BCYRN1 and HDAC11 mRNA expression levels was also identified in prostate tumor and healthy tissues. Therefore, the findings of the present study may provide novel insight into the effects of lncRNAs on prostate cancer, and may enable the development of new therapeutic methods for patients with prostate cancer.
\end{abstract}

\section{Introduction}

Prostate cancer is the second most commonly diagnosed type of cancer in men worldwide after lung cancer. In 2018, prostate cancer accounted for $13.5 \%$ of cancer cases in men, and was

Correspondence to: Professor Kaichen Wang, Department of Urology, China-Japan Union Hospital, Jilin University, 126 Xiantai Road, Changchun, Jilin 130033, P.R. China

E-mail:wangkaichen@aliyun.com

*Contributed equally

Key words: brain cytoplasmic RNA 1, histone deacetylase 11, prostate cancer responsible for $\sim 360,000$ deaths worldwide (1). Androgen deprivation therapy and chemotherapy, such as docetaxel, are currently the first-line treatment options for patients with metastatic prostate cancer $(2,3)$. However, patients my develop resistance to treatment, with ensuing cancer progression $(4,5)$. Therefore, understanding the complex signaling network involved in the development of prostate cancer is important for enabling the development of novel treatments for patients with prostate cancer.

Long non-coding RNAs (lncRNAs) are <200 nucleotides in length (6). Previous studies have demonstrated that lncRNAs may act as regulators of a variety of physiological processes $(7,8)$. Mechanistically, lncRNAs control gene expression at the epigenetic, transcriptional and post-transcriptional levels $(9,10)$. Previous studies have revealed that several lncRNAs can act as competing endogenous RNAs (ceRNAs), directly sponging microRNAs (miRNAs) via complementary sequence binding, and upregulating their miRNA targets (11). It has been demonstrated that lncRNAs play a pivotal role in prostate carcinogenesis $(12,13)$. LncRNA-LBCS was found to be downregulated in castration-resistant prostate cancer and it interacts with heterogeneous nuclear ribonucleoprotein $\mathrm{K}$ to suppress castration resistance of prostate cancer (14). Brain cytoplasmic RNA 1 (BCYRN1) is the shortest lncRNA, and it is 200 nucleotides in length. BCYRN1 was originally identified as a neuron-specific transcript, and has been shown to be overexpressed in several types of cancer (15). High expression of BCYRN1 has been found to be associated with poor prognosis of patients with colon cancer and esophageal squamous cell carcinoma $(16,17)$. In addition, the expression of BCYRN1 is a potential biomarker for breast cancer diagnosis, as abnormal expression of BCYRN1 is detected in serum from patients with breast cancer (18). However, the role of BCYRN1 in prostate cancer is currently unknown.

The histone deacetylase (HDAC) family is divided into classes I, IIa/b and IV, with 11 members, and class III, with 7 sirtuins (19). HDAC11 is a member of class IV HDACs (20). In contrast to class I HDACs, the role of HDAC11 in cancer remains elusive. Furthermore, depletion of HDAC11 inhibits metabolic activity in colorectal, prostate, ovarian and breast cancer cells (21). However, although abnormal expression of HDAC11 has been observed in tumors (21), the underlying molecular mechanism is unknown.

The aim of the present study was to investigate the role of BCYRN1 in prostate cancer progression and the involvement of HDAC11 in this process. 


\section{Materials and methods}

Clinical samples. A total of 72 pairs of prostate cancer and adjacent healthy prostate tissues were collected from patients with prostate cancer (aged 44-76 years), who underwent surgery at China-Japan Union Hospital between June 2014 and July 2018. None of the participants had received chemotherapy or radiotherapy prior to surgery. Written informed consent was obtained from all patients. The study was approved by and performed under the supervision of the Institutional Ethics Review Board of China-Japan Union Hospital.

Cell lines. The immortal prostate epithelial cell line RWPE-1, and the prostate cancer cell lines LNCaP (androgen-dependent prostate cancer), PC-3 (androgen-resistant prostate cancer) and DU145 (androgen-resistant prostate cancer) were purchased from the American Type Culture Collection. The cells were cultured in RPMI-1640 medium (Invitrogen; Thermo Fisher Scientific, Inc.) supplemented with 10\% FBS (Gibco; Thermo Fisher Scientific, Inc.) and maintained in a humid incubator with $5 \% \mathrm{CO}_{2}$ at $37^{\circ} \mathrm{C}$.

Cell transfection. Small interfering RNAs (siRNAs) targeting BCYRN1 (5'-CGCCTGTAATCCCAGCTCTCA-3') and control siRNA (5'-GGATACGGAGTACTATAGC-3') were synthesized and purchased from GenePharma. To knock down BCYRN1, BCYRN1 siRNA was transfected into PC-3 and DU145 cells using Lipofectamine RNAiMax (Invitrogen; Thermo Fisher Scientific, Inc.) following the manufacturer's protocol. The full length of HDAC11 was PCR-amplified from RWPE-1 cDNA, ligated into pcDNA3.1 and named pcDNA3.1-HDAC11. For overexpression of HDAC11, pcDNA3.1-HDAC11 was transfected into PC-3 and DU145 cells using Lipofectamine 3000 (Invitrogen; Thermo Fisher Scientific, Inc.) according to the manufacturer's protocol. miR-939-3p mimic (5'-CCC UGGGCCUCUGCUCCC CAG-3'), miR-939-3p inhibitor (5'-CUGGGGAGCAGAGGC CCAGGG-3'), miR-NC mimic (5'-AGGAUGUAUUACCAG UGAUCGG-3') and miR-NC inhibitor (5'-UCGAGACAC GUACGCAGAA-3') were purchased from RiboBio, Inc. miR-939-3p mimic, miR-939-3p inhibitor and their corresponding negative controls were transfected into PC-3 and DU145 cells using Lipofectamine 3000.

Protein extraction and western blotting. Protein lysates were prepared with RIPA lysis buffer (Beyotime Institute of Biotechnology). The protein concentration in the lysates was determined with a bicinchoninic acid protein assay kit (Pierce; Thermo Fisher Scientific, Inc.). Western blotting was performed using a standard procedure. In total, $20 \mu \mathrm{g}$ proteins were loaded in each lane of an $8 \%$ SDS gel and were separated by electrophoresis. Subsequently, the proteins were transferred to a PVDF membrane (Merck KGaA) and blocked in 5\% non-fat milk for $1 \mathrm{~h}$ at room temperature. The membrane was then incubated with primary antibody, followed by secondary antibody for $1 \mathrm{~h}$ at room temperature. The blots were developed with ECL Western Blotting Substrate (Pierce; Thermo Fisher Scientific, Inc.). For detection of H3, H4, H3K9ac and H4K16ac, the lysates were run on a $15 \%$ gel. Antibodies against H3 (cat. no. 61799, 1:5,000), H4 (cat. no. 61199, 1:5,000),
H3K9ac (cat. no. 39917, 1:2,000) and H4K16ac (cat. no. 61529, 1:2,000) were purchased from Active Motif. Antibodies against HDAC11 (cat. no. 58442, 1:1,000) and $\beta$-actin (cat. no. 3700, 1:5,000) were purchased from Cell Signaling Technology, Inc. HRP-conjugated secondary antibodies against rabbit (cat. no. ab150077, 1:10,000) and mouse (cat. no. ab205719, 1:10,000) were obtained from Abcam.

$R N A$ extraction and reverse transcription-quantitative $P C R$ (RT-qPCR) analysis. RNA was extracted from cells and specimens using TRIzol reagent (Invitrogen; Thermo Fisher Scientific, Inc.) following the manufacturer's protocol. The concentration and quality of RNA were measured using NanoDrop 2000 (Thermo Fisher Scientific, Inc.). RNA was reverse-transcribed into first-strand cDNA with a PrimeScript RT reagent kit (Takara Bio, Inc.) at $37^{\circ} \mathrm{C}$ for $15 \mathrm{~min}$ and at $85^{\circ} \mathrm{C}$ for $5 \mathrm{sec}$. qPCR was performed with SYBR Premix Ex Taq (Takara Bio, Inc.) with the following conditions: Initial denaturation at $95^{\circ} \mathrm{C}$ for $5 \mathrm{sec}$, followed by 35 cycles at $94^{\circ} \mathrm{C}$ for $15 \mathrm{sec}$ and $55^{\circ} \mathrm{C}$ for $30 \mathrm{sec} . \beta$-actin and U6 were used as internal controls for mRNA and lncRNA, and miRNA, respectively. The relative expression level was calculated by the $2^{-\Delta \Delta \mathrm{Cq}}$ method (22). The primer sequences were as follows: BCYRN1, forward 5'-CTGGGCAATATAGCGAGAC-3' and reverse 5'-TGCTTTGAGGGAAGTTACG-3'; stem-loop primer, 5'-CTCAACTGGTGTCGTGGAGTCGGCAATTCA GTTGAGCTGGGAGCUCCCCAG-3'; miR-939-3p, forward 5'-GCCGAGCCCTGGGCCTCTGC-3' and reverse 5'-CTC AACTGGTGTCGTGGA-3'; U6, forward 5'-ATTGGAACG ATACAGAGAAGATT-3' and reverse 5'-GGAACGCTTCAC GAATTTG-3'; HDAC11, forward 5'-ACCCAGACAGGA GGAACCATA-3' and reverse 5'-TGATGTCCGCATAGG CACAG-3'; cyclin-dependent kinase inhibitor 1A (CDKN1A), forward 5'-TGTCCGTCAGAACCCATGC-3' and reverse 5'-AAAGTCGAAGTTCCATCGCTC-3'; optineurin (OPTN), forward 5'-CCAAACCTGGACACGTTTACC-3' and reverse 5'-CCTCAAATCTCCCTTTCATGGC-3'; glucose transporter 1 (GLUT1), forward 5'-GGCCAAGAGTGTGCTAAAGAA-3' and reverse 5'-ACAGCGTTGATGCCAGACAG-3'; and $\beta$-actin, forward 5'-CATGTACGTTGCTATCCAGGC-3' and reverse 5'-CTCCTTAATGTCACGCACGAT-3'.

Cell proliferation assay. The Cell Counting Kit-8 assay (CCK-8 kit; DoJinDo Molecular Technologies, Inc.) was used to detect cell proliferation ability. Briefly, 2,000 cells were seeded in each well of a 96-well plate. Subsequently, $10 \mu \mathrm{l}$ CCK-8 solution was added into the culture medium in the wells at $0,1,2$, 3,4 and 5 days after transfection of siRNA and/or plasmid. After incubation for $2 \mathrm{~h}$ at $37^{\circ} \mathrm{C}$, the medium containing CCK-8 solution was transferred to a new 96-well plate, and the absorbance at $450 \mathrm{~nm}$ was measured by a microplate reader (Bio-Rad Laboratories, Inc.) to reflect cell proliferation ability.

Flow cytometry analysis. Cell apoptosis was determined by flow cytometry analysis with an Annexin V-FITC-PI Apoptosis Detection kit (Invitrogen; Thermo Fisher Scientific, Inc.). Briefly, cells were harvested and suspended in Annexin V Binding Buffer. Annexin V-FITC and PI staining solution were added to the cells and incubated at room temperature for $10 \mathrm{~min}$. Subsequently, the cells were subjected to flow 
cytometry analysis on a MACSQuant system (Miltenyi Biotec). Data were analyzed using FlowJo software v10.6.1 (Becton, Dickinson and Company).

Glucose uptake and lactate production assay. A glucose uptake fluorometric assay kit (BioVision Research, Inc.) was applied to detect glucose uptake in PC-3 and DU145 cells. Cells were seeded at a density of 2,000/well in 96-well plates. Subsequently, the cells were starved overnight. Next, $1 \mu \mathrm{M}$ insulin was added, followed by the addition of $1 \mathrm{mM}$ 2-deoxy-D-glucose (2-DG). A mixture containing Picoprobe was added to the medium. The optical density (OD) value was then determined by a microplate reader (Bio-Rad Laboratories, Inc.) to reflect 2-DG uptake. Lactate production was detected using a lactic acid assay kit (Sigma Aldrich; Merck KGaA). The OD value of treated cells was applied to the standard curve to calculate lactate production levels.

Determination of HDAC activity. The activity of HDACs in PC-3 and DU145 cells was detected with a colorimetric HDAC activity assay kit (BioVision Research, Inc.), following the manufacturer's instructions. The relative OD value at $405 \mathrm{~nm}$ was analyzed with an iMark Microplate Absorbance Reader (Bio-Rad Laboratories, Inc.) to reflect the activity of HDACs.

Dual-luciferase reporter assay. The full length of the 3' untranslated region (UTR) of BCYRN1 and HDAC11 was amplified from RWPE-1 cDNA, and ligated into pGL3-basic plasmids. Mutations were introduced into BCYRN1 and HDAC11 3'UTR with a Quick Site-directed Mutation kit (Agilent Technology; Thermo Fisher Scientific, Inc.). Plasmids and miR-939-3p mimic were co-transfected into cells using Lipofectamine 3000 (Invitrogen; Thermo Fisher Scientific, Inc.) following the manufacturer's protocol. After 2 days, the relative luciferase activity of each well was measured with a Dual Luciferase Reporter Assay system (Promega Corporation). Firefly luciferase activity was normalized to Renilla luciferase activity.

Statistical analysis. All data were analyzed using GraphPad Prism 6 (GraphPad Software, Inc.), and are presented as the mean \pm standard deviation. The correlation between BCYRN1 and HDAC11 mRNA expression levels was examined by Pearson's correlation analysis. Comparison of two groups was conducted with a Student's t-test, while three or more groups were compared with one-way ANOVA followed by Newman-Keuls analysis. The association between BCYRN1 expression and the characteristics of patients with prostate cancer was analyzed with the $\chi^{2}$ test. All P-values were two-tailed and $\mathrm{P}<0.05$ was considered to indicate a statistically significant difference.

\section{Results}

BCYRN1 is upregulated inprostate cancer. Bioinformatics analysis of The Cancer Genome Atlas-Prostate Adenocarcinoma (TCGA-PRAD) dataset (52 healthy prostate tissues and 499 prostate tumors) demonstrated that the expression of BCYRN1 was significantly upregulated in prostate tumors (Fig. 1A). To
Table I. Association between BCYRN1 levels and the characteristics of patients with prostate cancer.

\begin{tabular}{lcccc}
\hline & \multicolumn{3}{c}{$\begin{array}{c}\text { BCYRN1 } \\
\text { expression }\end{array}$} \\
\cline { 3 - 4 } Characteristics & Total & High & Low & P-value \\
\hline Age (years) & & & & 0.809 \\
$\quad \leq 55$ & 28 & 13 & 15 & \\
$>55$ & 44 & 23 & 21 & \\
Stage & & & & 0.341 \\
I-II & 31 & 13 & 18 & \\
III-IV & 41 & 23 & 18 & \\
Gleason score & & & & 0.004 \\
$\leq 4$ & 20 & 4 & 16 & \\
$5-7$ & 32 & 18 & 14 & \\
$8-10$ & 20 & 14 & 6 & \\
Serum PSA (ng/ml) & & & & 0.406 \\
$\quad \leq 4$ & 15 & 6 & 9 & \\
$>4$ and $\leq 10$ & 34 & 16 & 18 & \\
$>10$ & 23 & 14 & 9 & \\
\hline
\end{tabular}

BCYRN1, brain cytoplasmic RNA 1; PSA, prostate-specific antigen.

verify this result, BCYRN1 expression was detected in 72 pairs of prostate tumors and matched healthy tissues. The RT-qPCR results indicated that BCYRN1 was overexpressed in prostate tumors (Fig. 1B). Moreover, it was observed that BCYRN1 was significantly upregulated in prostate tumors with a high Gleason score (8-10) compared with those with an intermediate Gleason score (5-7). Furthermore, tumors with a low Gleason score (2-4) exhibited a lower expression of BCYRN1 compared with those with an intermediate Gleason score (5-7) (Fig. 1C). The expression of BCYRN1 was not found to be associated with age, serum prostate-specific antigen concentration or stage in patients with prostate cancer (Table I). Therefore, these results suggested that high expression of BCYRN1 is associated with prostate cancer progression. The expression of BCYRN1 in androgen-dependent prostate cancer cell lines (PC-3 and DU145), an androgen-sensitive prostate cancer cell line (LNCaP) and an immortal prostate epithelial cell line (RWPE-1) was also examined. BCYRN1 was found to be overexpressed in prostate cancer cells, particularly the androgen-resistant prostate cancer cell lines PC-3 and DU145, compared with RWPE-1 cells (Fig. 1D).

Knockdown of BCYRN1 inhibits cell proliferation and glucose metabolism, and induces apoptosis in prostate cancer cells. To investigate the role of BCYRN1 in prostate cancer, BCYRN1 siRNA was transfected into prostate cancer cells. It was observed that transfection of BCYRN1 siRNA significantly decreased BCYRN1 expression in PC-3 and DU145 cells (Fig. 2A). Moreover, the cell proliferation assay revealed that knockdown of BCYRN1 significantly inhibited the proliferation of PC-3 and DU145 cells (Fig. 2B and C). Furthermore, the flow cytometry analysis results demonstrated 
A
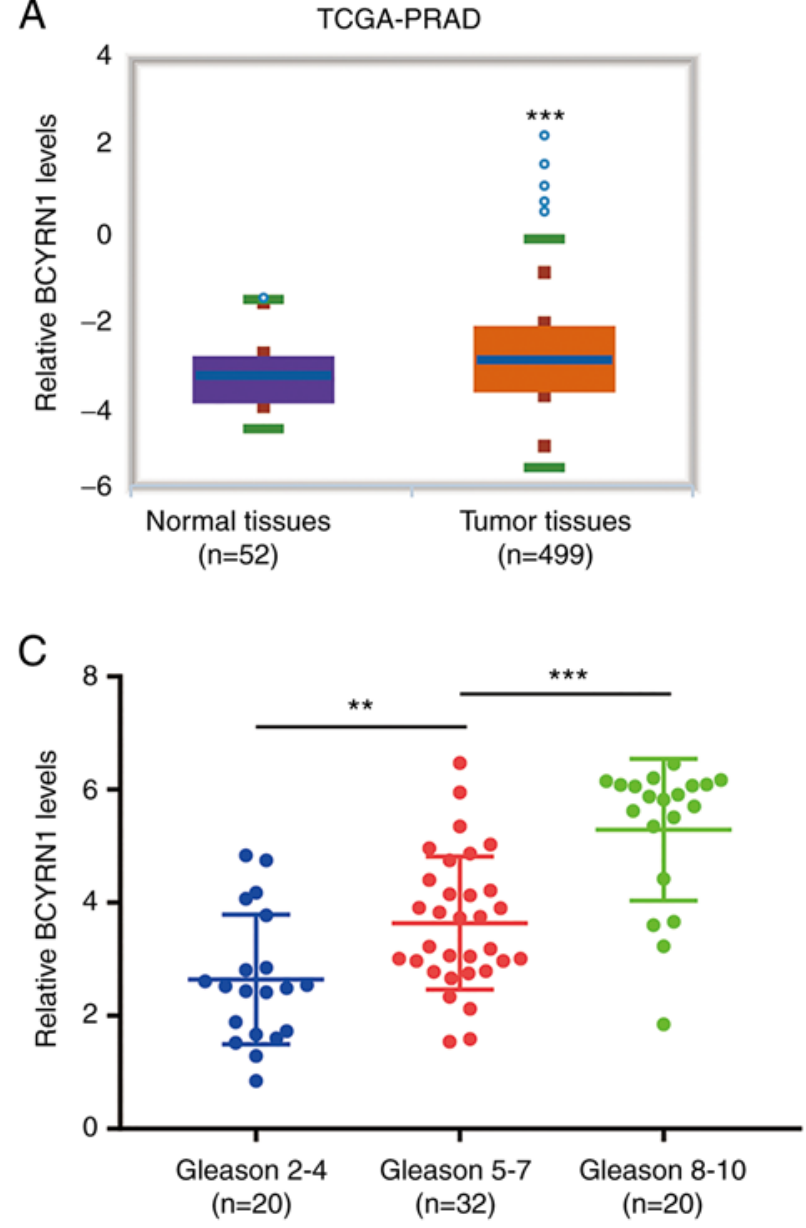

B

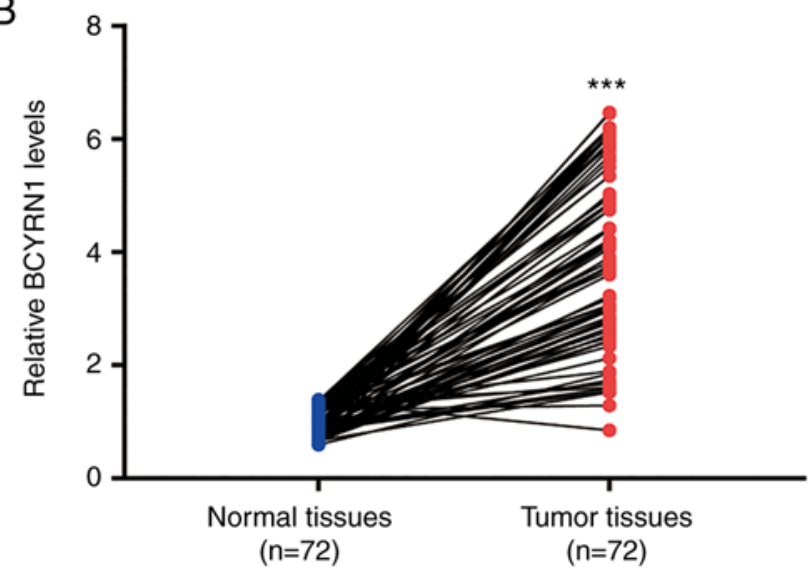

D

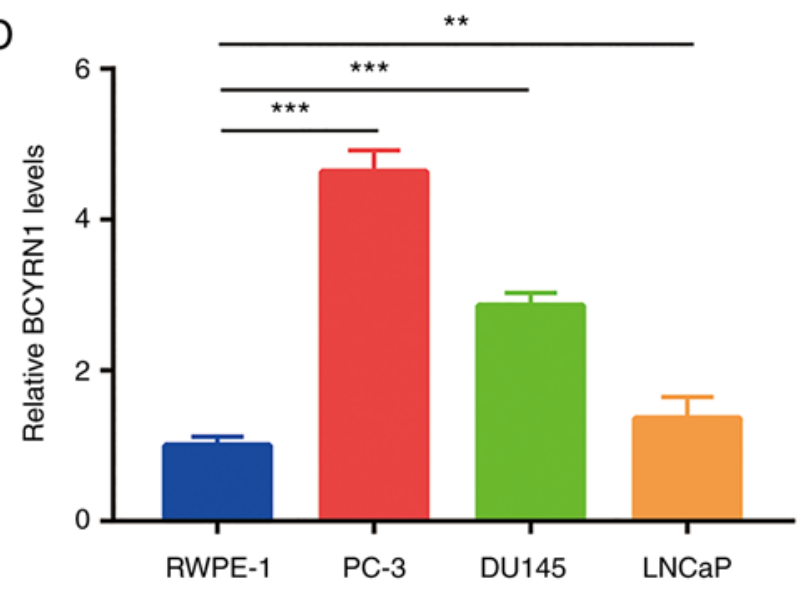

Figure 1. Upregulation of BCYRN1 in prostate cancer. (A) The expression of BCYRN1 in The Cancer Genome Atlas-Prostate Adenocarcinoma dataset was analyzed with StarBase V3. BCYRN1 was significantly upregulated in prostate tumors $(\mathrm{n}=499)$ compared with normal prostate $(\mathrm{n}=52)$. (B) Reverse transcription-quantitative PCR revealed that the expression of BCYRN1 was increased in prostate tumors $(\mathrm{n}=72)$ compared with matched normal tissues. (C) Expression of BCYRN1 in prostate tumors of high (8-10), intermediate (5-7) and low (2-4) Gleason score. (D) BCYRN1 was increased in androgen-resistant prostate cancer cells (PC-3 and DU145) and androgen-dependent prostate cancer cells (LNCaP) compared with immortal prostate epithelial cells (RWPE-1). ${ }^{* *} \mathrm{P}<0.01 ;{ }^{* * *} \mathrm{P}<0.001$. BCYRN1, brain cytoplasmic RNA 1; TCGA-PRAD, The Cancer Genome Atlas-Prostate Adenocarcinoma.

that BCYRN1 knockdown induced apoptosis of PC-3 and DU145 cells (Fig. 2D and E). Abnormal glucose metabolism is crucial for prostate cancer progression. The glucose uptake assay results suggested that BCYRN1 silencing inhibited the glucose uptake ability of PC-3 and DU145 cells (Fig. 2F). In addition, BCYRN1 silencing reduced the levels of lactate produced by PC-3 and DU145 cells, thereby indicating that glucose metabolism in PC-3 and DU145 cells is promoted by BCYRN1 (Fig. 2G). GLUT1 is crucial for glucose metabolism in prostate cancer cells (23). RT-qPCR analysis revealed that BCYRN1 silencing decreased GLUT1 mRNA levels in PC-3 and DU145 cells (Fig. 2H).

BCYRN1 binds to miR-939-3p and represses its expression in prostate cancer cells. According to the prediction results of miRDB, five miRNAs harbored putative binding sites for BCYRN1 (Fig. 3A). Among those, bioinformatics analysis of prostate cancer datasets (GSE65061 and GSE46738) revealed that miR-939-3p was downregulated in prostate cancer (24). Furthermore, the RT-qPCR results indicated that silencing of BCYRN1 upregulated miR-939-3p in PC-3 and DU145 cells (Fig. 3B). In the present study, miR-939-3p was overexpressed in prostate cancer cells via transfection of miR-939-3p mimic (Fig. 3C). It was observed that elevation of miR-939-3p decreased BCYRN1 expression in PC-3 and DU145 cells (Fig. 3D). However, transfection with a miR-939-3p inhibitor decreased miR-939-3p expression, which was accompanied by an elevation of BCYRN1 expression in prostate cancer cells (Fig. 3E and F). It was also observed that overexpression of miR-939-3p decreased the relative luciferase activity of BCYRN1-wild-type (WT), but not BCYRN1-mutant (Mut), which contained mutations in the putative binding site. Therefore, these results suggested that miR-939-3p may directly bind to BCYRN1 in PC-3 cells (Fig. 3G). Moreover, miR-939-3p overexpression reduced the relative luciferase activity of BCYRN1-WT in DU145 cells (Fig. 3H). Collectively, these results indicate that BCYRN1 binds to miR-939-3p and represses its expression in prostate cancer cells.

HDAC11 is directly repressed by miR-939-3p. According to the ceRNA hypothesis, miRNA regulates gene expression via binding to target gene mRNA (25). In the present study, TargetScan (http://www.targetscan.org/vert_72/) predicted HDAC11 as a potential target gene of miR-939-3p (Fig. 4A). 

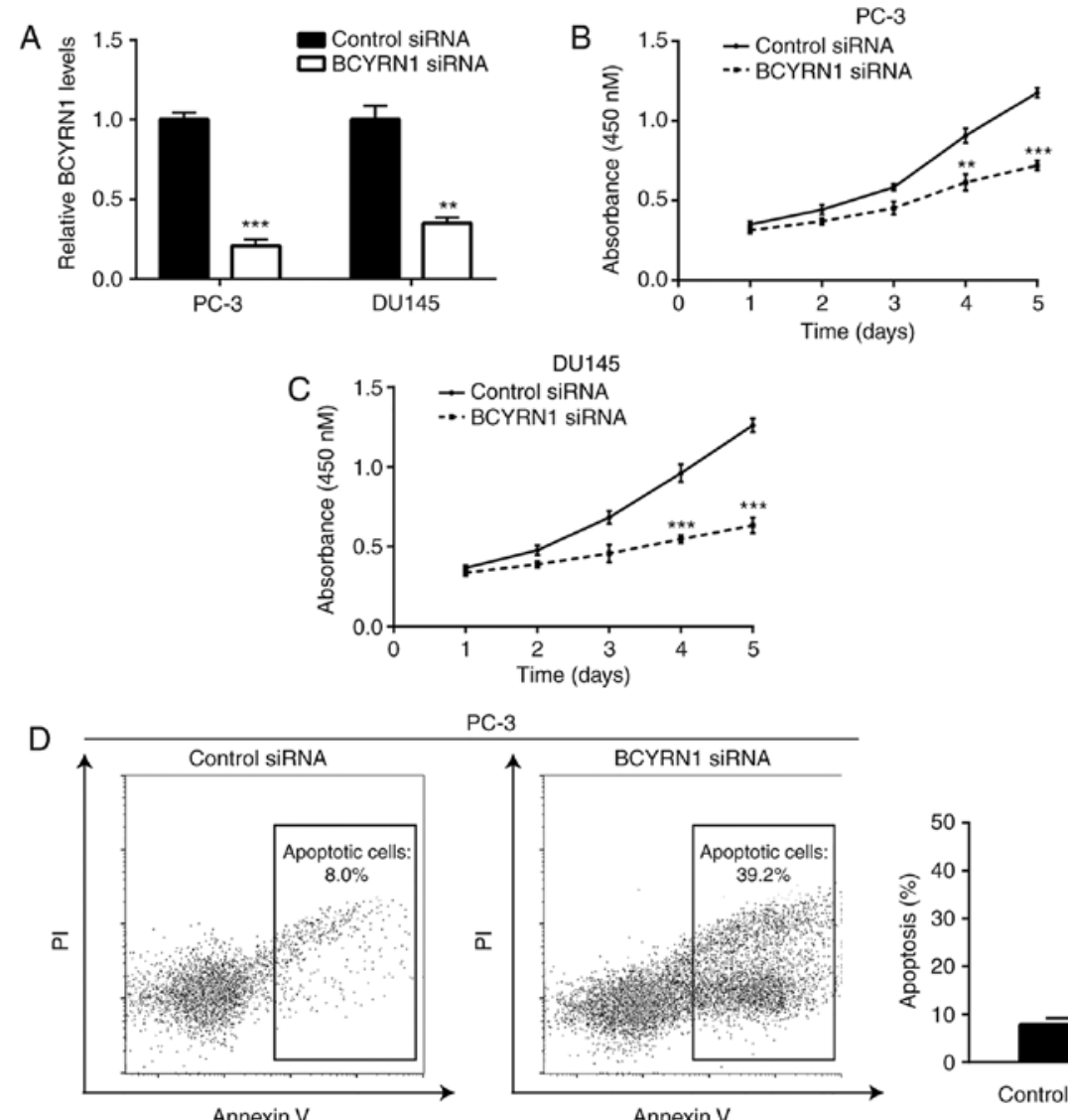

PC-3 BCYRN1 SiRNA
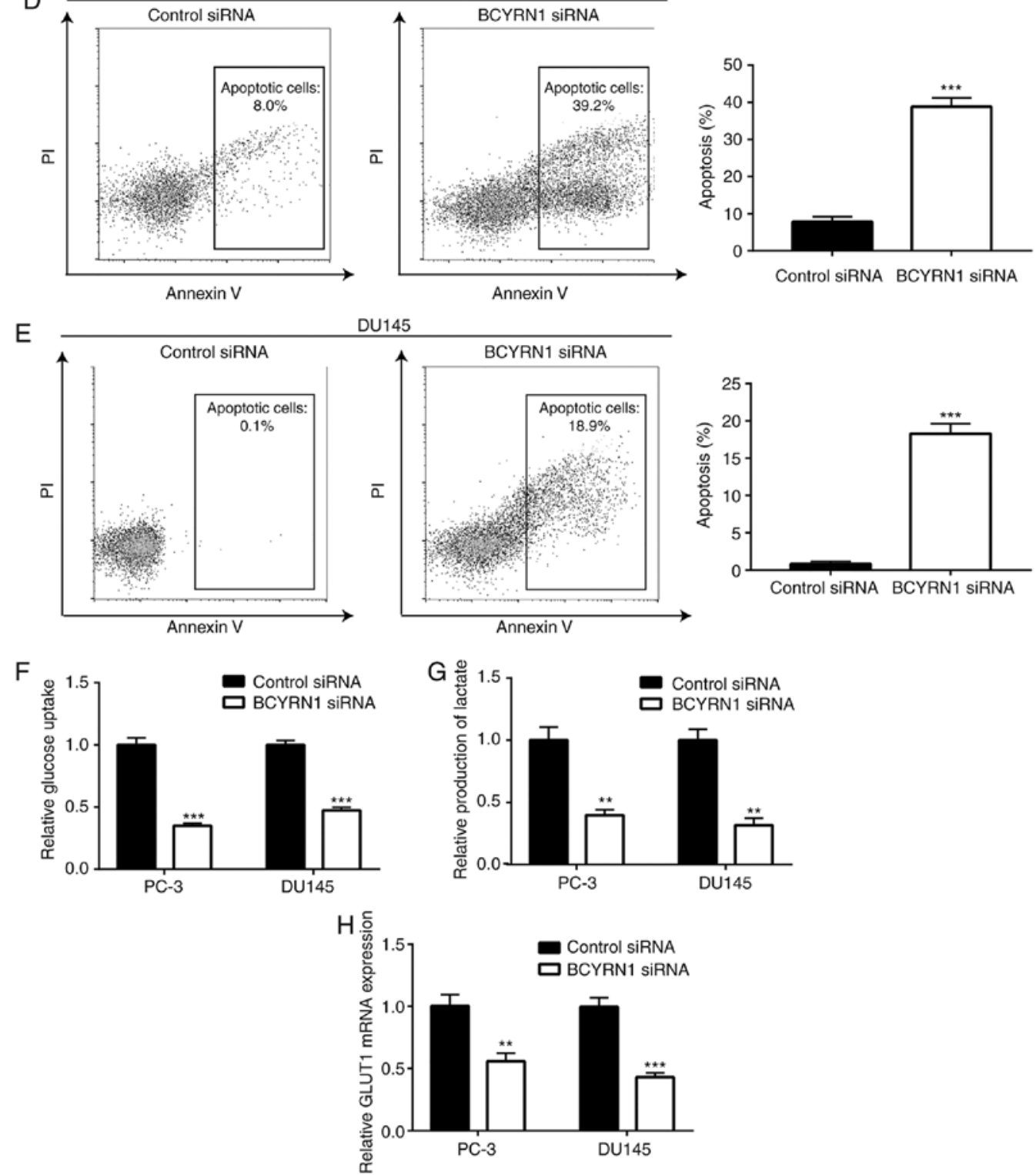

Figure 2. BCYRN1 silencing inhibited proliferation and glucose metabolism and induced apoptosis in prostate cancer cells. (A) Transfection of BCYRN1 siRNA decreased BCYRN1 expression in PC-3 and DU145 cells. (B) BCYRN1 silencing inhibited the proliferation of PC-3 cells. (C) BCYRN1 silencing inhibited the proliferation of DU145 cells. (D) BCYRN1 silencing induced apoptosis of PC-3 cells. (E) BCYRN1 silencing induced apoptosis of DU145 cells. (F) Silencing of BCYRN1 inhibited glucose uptake in PC-3 and DU145 cells. (G) Silencing of BCYRN1 inhibited lactate production in PC-3 and DU145 cells. (H) Silencing of BCYRN1 reduced GLUT1 mRNA expression in PC-3 and DU145 cells. ${ }^{* *} \mathrm{P}<0.01$; ${ }^{* * *} \mathrm{P}<0.001$. BCYRN1, brain cytoplasmic RNA 1; GLUT1, glucose transporter 1. 
A

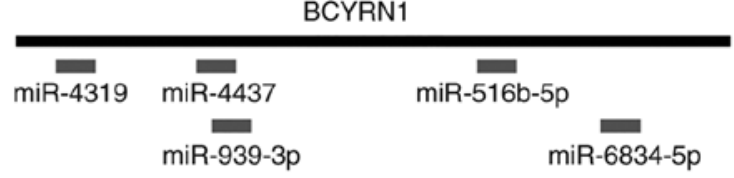

BCYRN1-WT 5'-GGAGGATAGCTTGAGCCCAGGA-3'

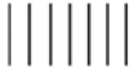

miR-939-3p $\quad 3^{\prime}$-GACCCCUCGUCUCCGGGUCCC-5

BCYRN1-Mut 5'-GGAGGATAGCTTGAGCAAAGGA-3'

C

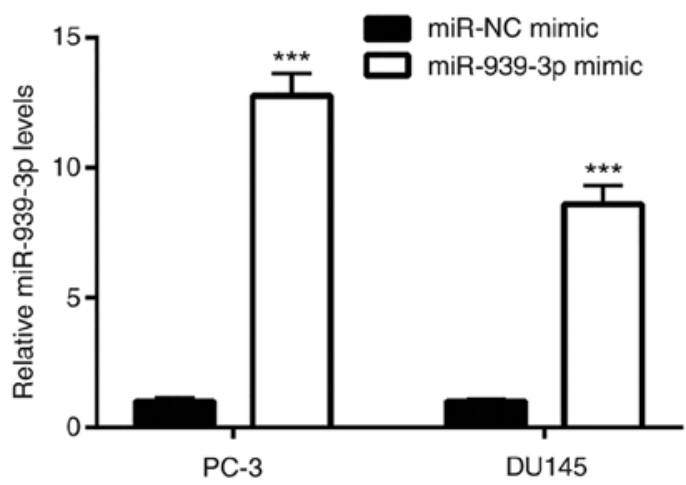

E
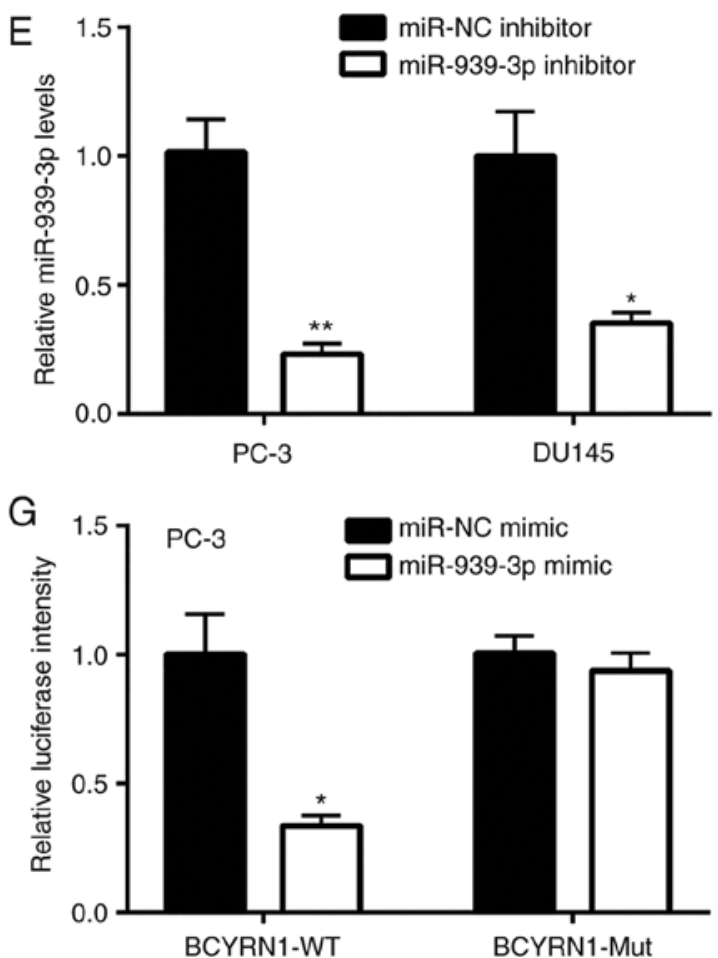

B

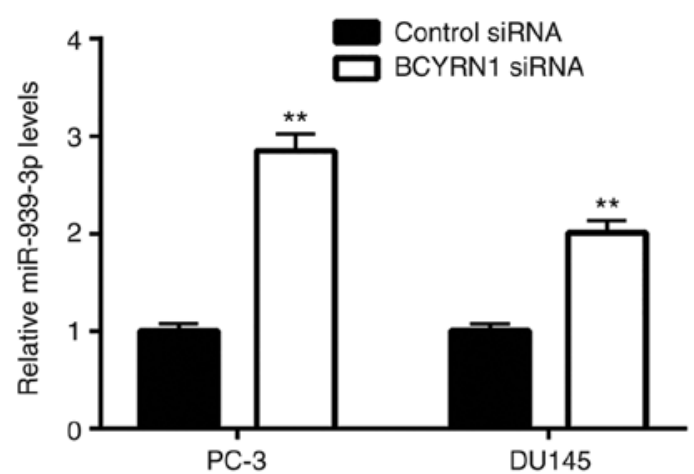

$\left.{ }^{1.5}\right]$ miR-NC mimic

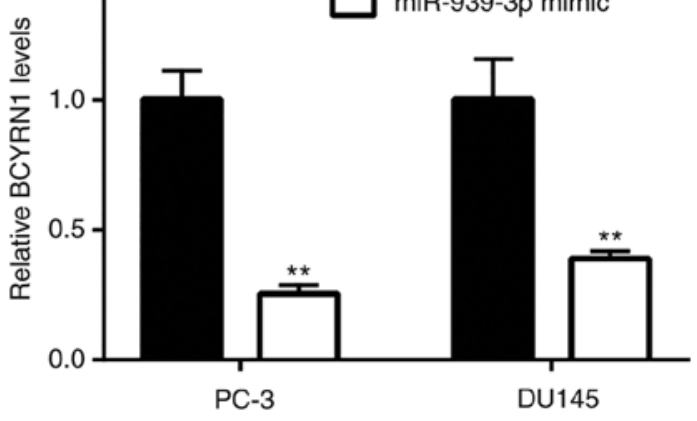

F 2.5 miR-NC inhibitor

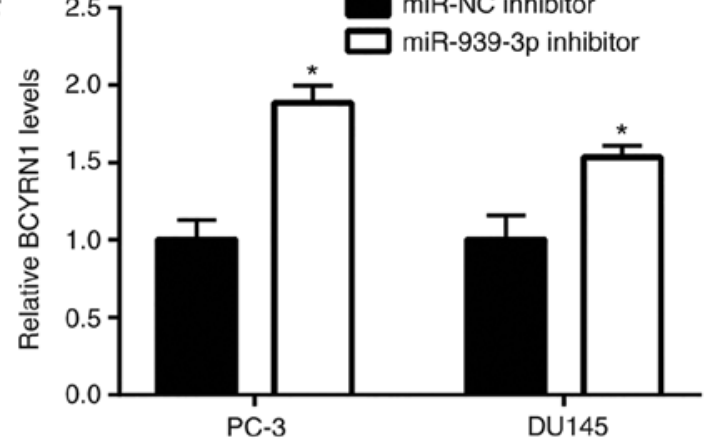

$\mathrm{H}$

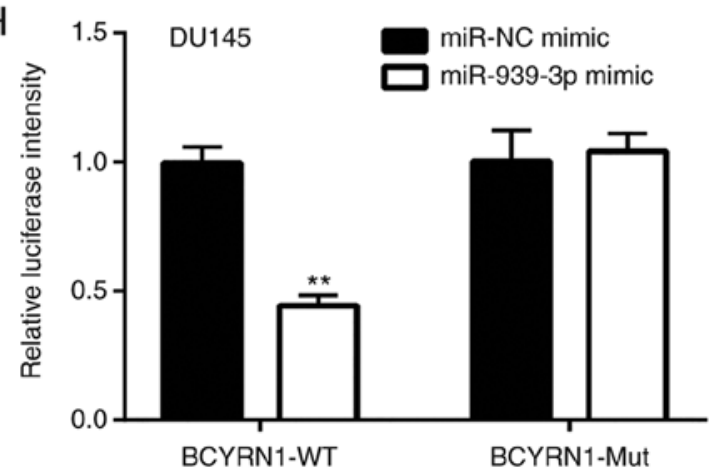

Figure 3. BCYRN1 sponged miR-939-3p in prostate cancer cells. (A) There were putative binding sites for miR-4319, miR-4437, miR-939-3p, miR-516b-5p and miR-6834-5p on the BCYRN1 sequence. (B) Silencing of BCYRN1 increased miR-939-3p levels in PC-3 and DU145 cells. (C) Transfection of miR-939-3p mimic elevated miR-939-3p expression in PC-3 and DU145 cells. (D) BCYRN1 expression was decreased in PC-3 and DU145 cells transfected with miR-939-3p mimic. (E) Transfection of miR-939-3p inhibitor decreased miR-939-3p expression in PC-3 and DU145 cells. (F) Downregulation of miR-939-3p increased BCYRN1 expression in PC-3 and DU145 cells. (G) Overexpression of miR-939-3p reduced the relative luciferase activity of pGL3-BCYRN1 in PC-3 cells. (H) Overexpression of miR-939-3p reduced the relative luciferase activity of pGL3-BCYRN1 in DU145 cells. ${ }^{*} \mathrm{P}<0.05 ;{ }^{* *} \mathrm{P}<0.01 ;{ }^{* * *} \mathrm{P}<0.001$. BCYRN1, brain cytoplasmic RNA 1 .

Furthermore, overexpression of miR-939-3p decreased HDAC11 mRNA expression in PC-3 and DU145 cells (Fig. 4B). Western blotting demonstrated that miR-939-3p overexpression reduced HDAC11 protein expression in prostate cancer cells
(Fig. 4C). Luciferase plasmids containing HDAC11 3'UTR-WT and -Mut were used to study the association between HDAC11 and miR-939-3p (Fig. 4D). Overexpression of miR-939-3p reduced the relative luciferase activity of HDAC11 3'UTR-WT, 
A

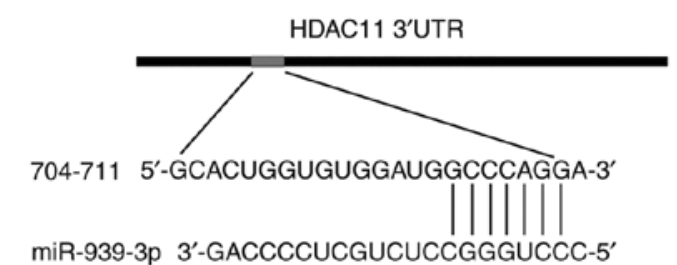

C

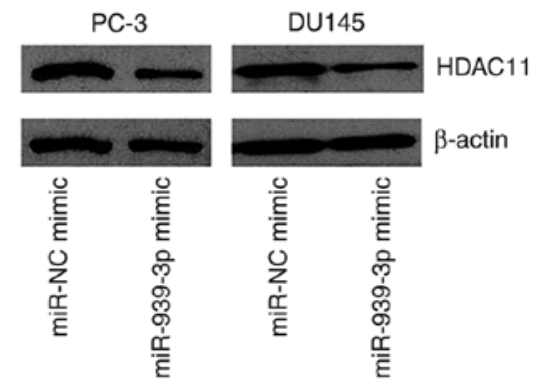

D
B
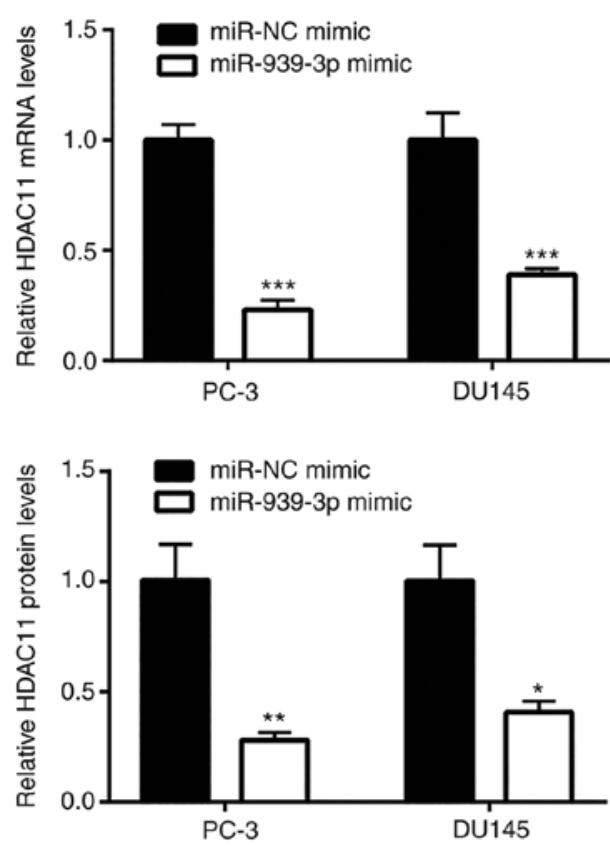

E

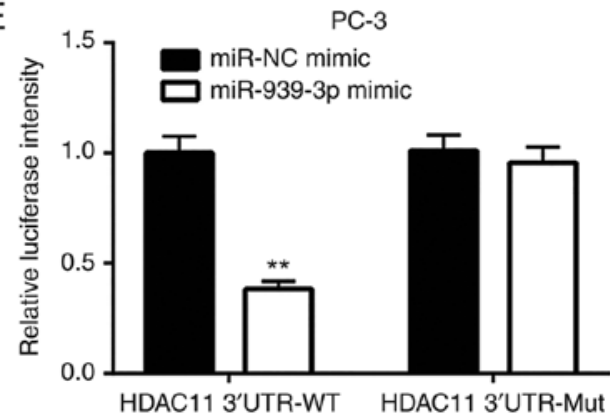

G

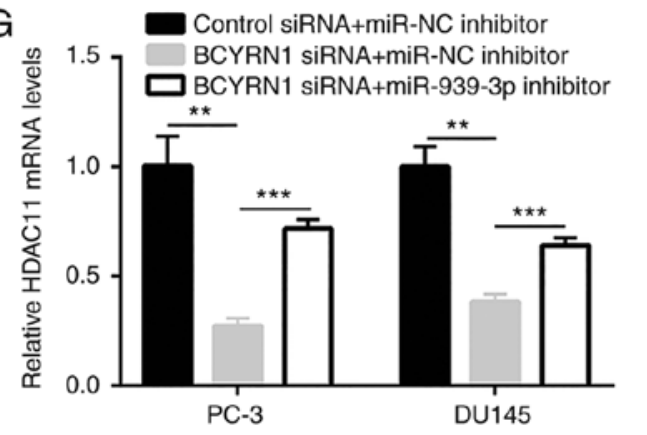

Control siRNA+miR-NC inhibitor
$\mathrm{H}$

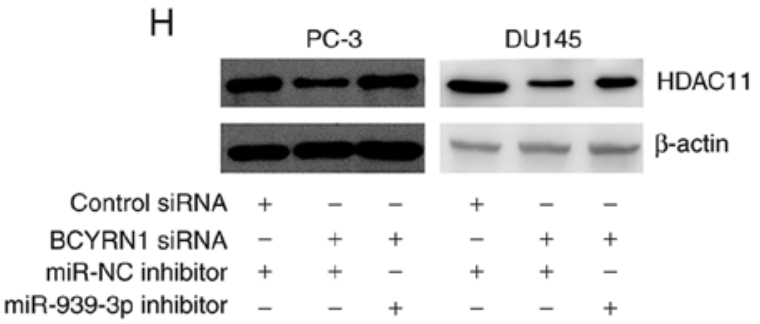

Control siRNA BCYRN1 siRNA miR-NC inhibitor miR-939-3p inhibitor

DU145

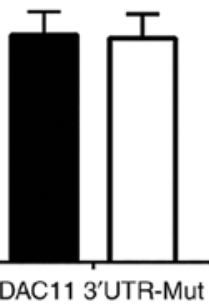
BCYRN1 siRNA+miR-NC inhibitor

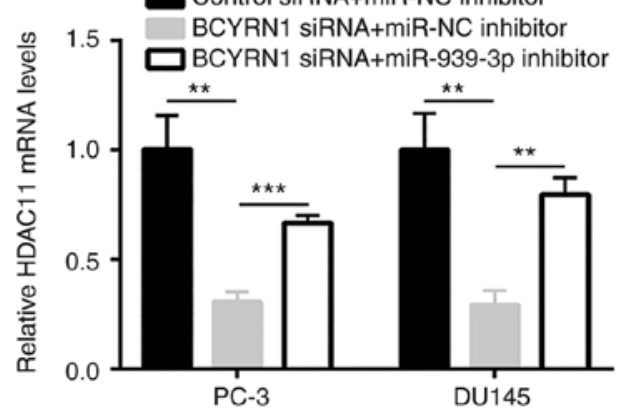

Figure 4. BCYRN1 upregulated HDAC11 via sponging miR-939-3p. (A) There was a putative binding site for miR-939-3p on HDAC11 3'UTR.(B) Overexpression of miR-939-3p decreased HDAC11 mRNA expression in PC-3 and DU145 cells. (C) Western blotting revealed that overexpression of miR-939-3p decreased HDAC11 protein expression in PC-3 and DU145 cells. (D) Sequence alignment of miR-939-3p with HDAC11 3'UTR-WT and HDAC11 3'UTR-Mut. (E) Overexpression of miR-939-3p decreased the relative luciferase activity of HDAC11 3'UTR-WT in PC-3 cells. (F) Overexpression of miR-939-3p decreased the relative luciferase activity of HDAC11 3'UTR-WT in DU145 cells. (G) Silencing of BCYRN1 decreased HDAC11 mRNA expression, and this effect was reversed following transfection of miR-939-3p inhibitor in PC-3 and DU145 cells. (H) Silencing of BCYRN1 decreased HDAC11 protein expression, and this effect was reversed following transfection of miR-939-3p inhibitor in PC-3 and DU145 cells. ${ }^{*} \mathrm{P}<0.05 ;{ }^{* *} \mathrm{P}<0.01 ;{ }^{* * *} \mathrm{P}<0.001$. BCYRN1, brain cytoplasmic RNA 1; HDAC11, histone deacetylase 11; UTR, untranslated region; WT, wild-type; Mut, mutant. 
A
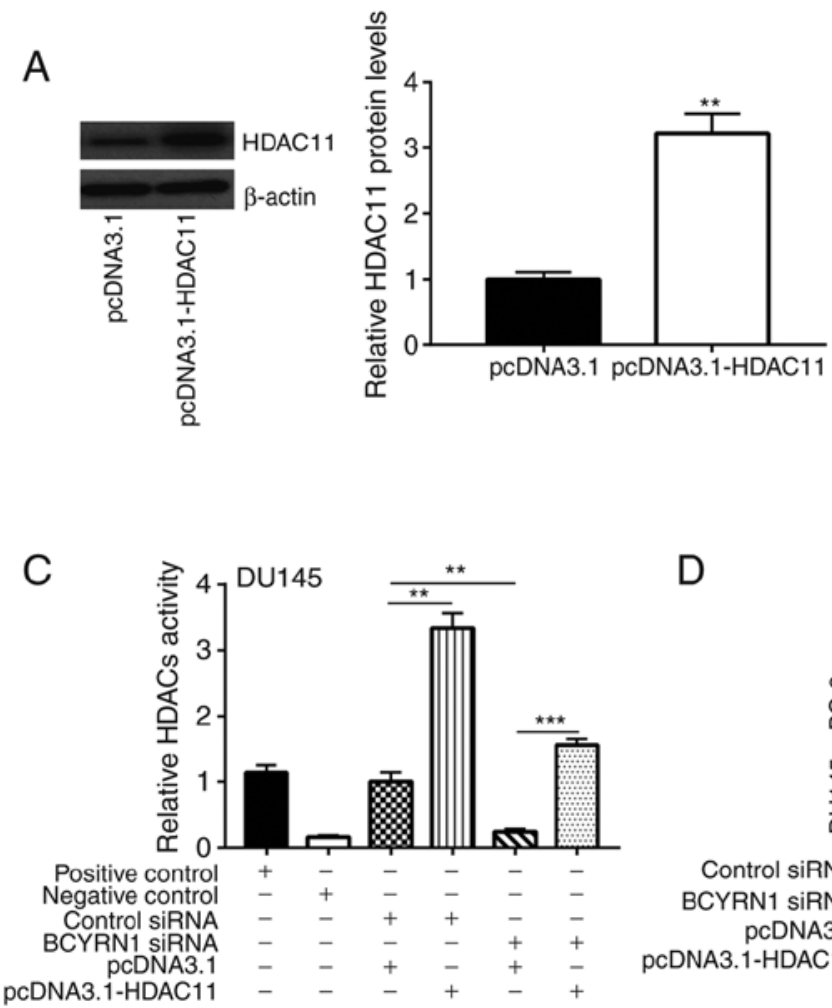

D
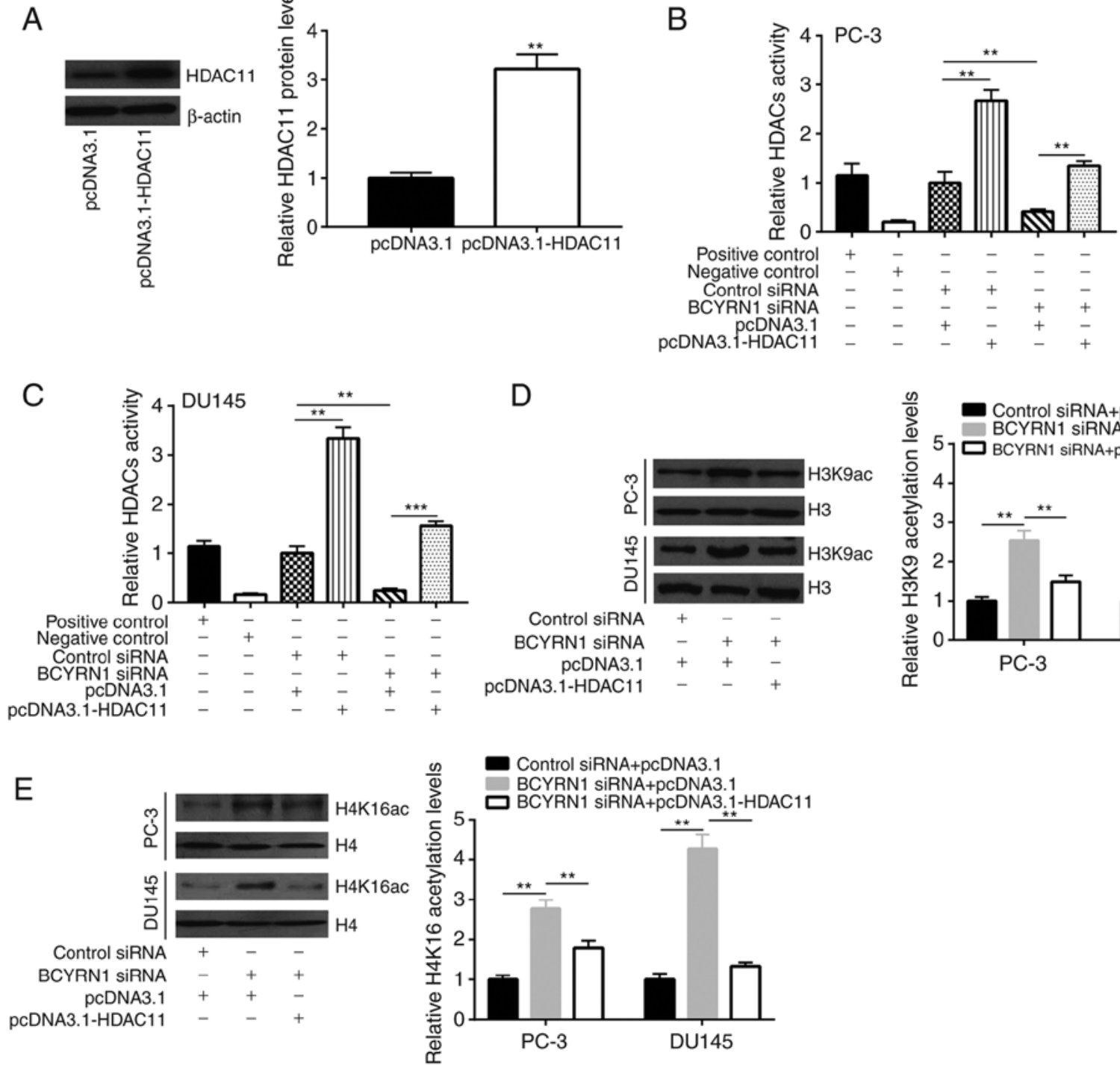

$\mathrm{F}$
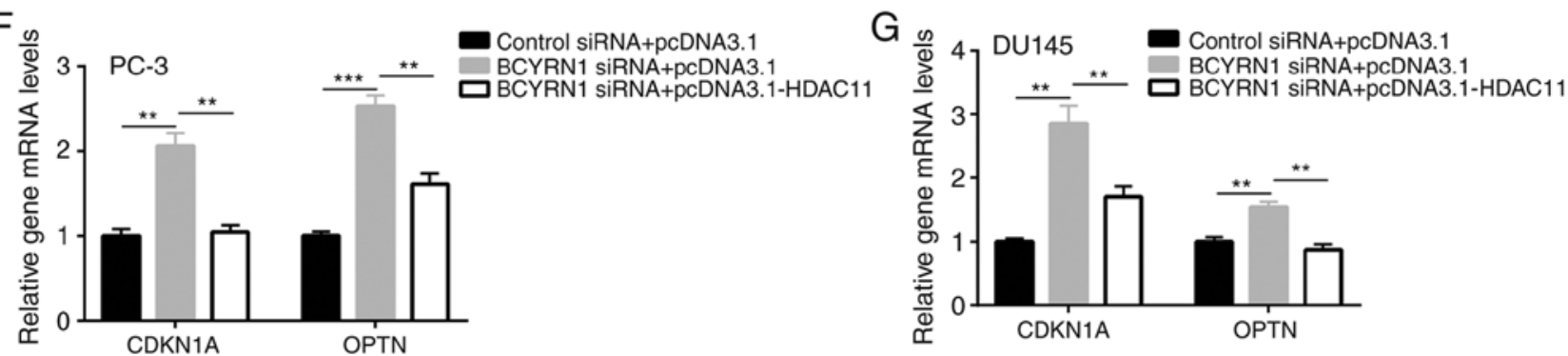

Figure 5. BCYRN1 decreased global acetylation of H3K9 and H4K16 via regulating HDAC11 expression. (A) Transfection of recombinant HDAC11 increased HDAC11 expression in PC-3 cells. (B) Overexpression of HDAC11 increased HDAC activity, while silencing of BCYRN1 decreased HDAC activity in PC-3 cells. (C) Overexpression of HDAC11 increased HDAC activity, while silencing of BCYRN1 decreased HDAC activity in DU145 cells. (D) Silencing of BCYRN1 increased global acetylation levels of H3K9, and HDAC11 overexpression reversed the elevation of H3K9 acetylation in PC-3 and DU145 cells. (E) Silencing of BCYRN1 increased global acetylation levels of H4K16, and HDAC11 overexpression reversed the elevation of H4K16 acetylation in PC-3 and DU145 cells. (F) Silencing of BCYRN1 increased CDKN1A and OPTN mRNA expression, and HDAC11 overexpression reversed the elevation of CDKN1A and OPTN mRNA expression in PC-3 cells. (G) Silencing of BCYRN1 increased CDKN1A and OPTN mRNA expression, and HDAC11 overexpression reversed the elevation of CDKN1A and OPTN mRNA expression in DU145 cells. ${ }^{* *} \mathrm{P}<0.01 ;{ }^{* * * *} \mathrm{P}<0.001$. BCYRN1, brain cytoplasmic RNA 1 ; HDAC11, histone deacetylase 11; CDKN1A, cyclin-dependent kinase inhibitor 1A; OPTN, optineurin.

but not HDAC11 3'UTR-Mut in PC-3 cells (Fig. 4E). Moreover, a similar result was observed in DU145 cells (Fig. 4F). In addition, silencing of BCYRN1 decreased HDAC11 mRNA expression, which was reversed by miR-939-3p inhibitor in PC-3 and DU145 cells (Fig. 4G). At the protein expression level,
BCYRN1 knockdown reduced HDAC11 expression, which was reversed by miR-939-3p inhibitor (Fig. 4H).

BCYRN1 represses tumor suppressor expression via upregulation of HDAC11 and deacetylation of H3K9 and 
A
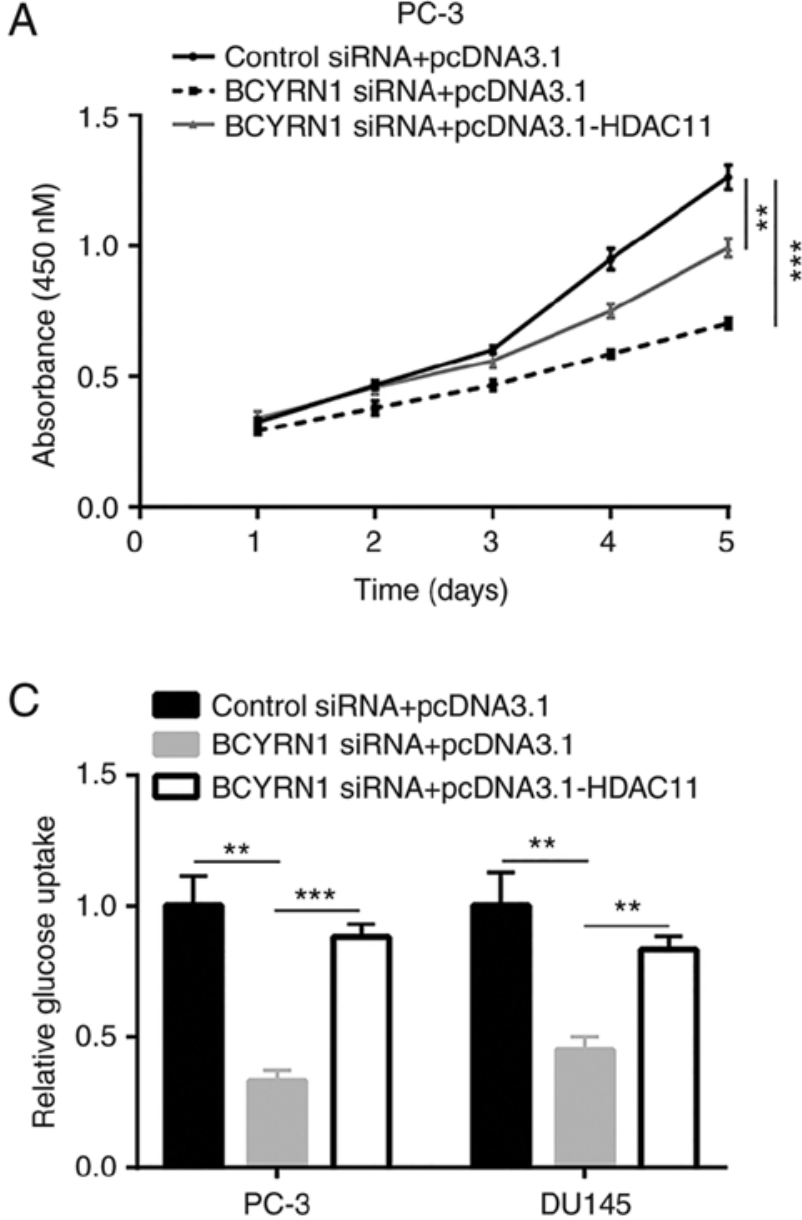

B

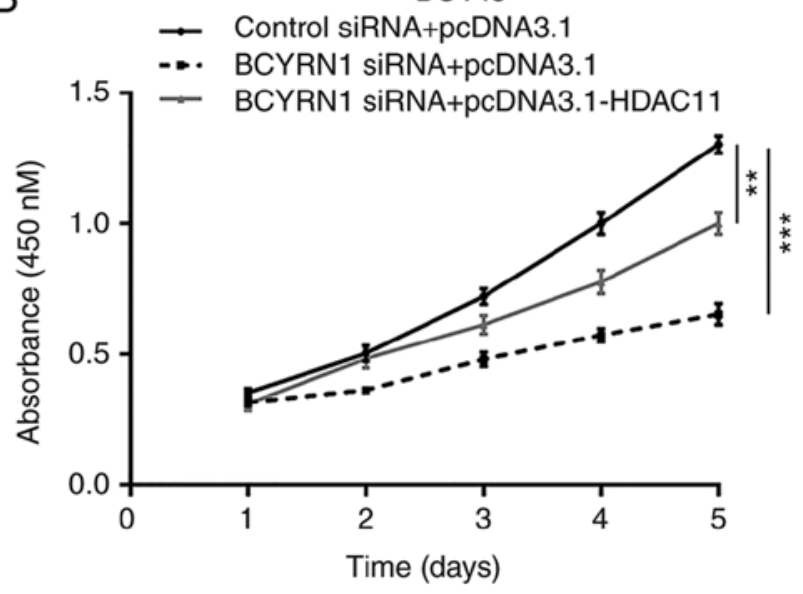

D

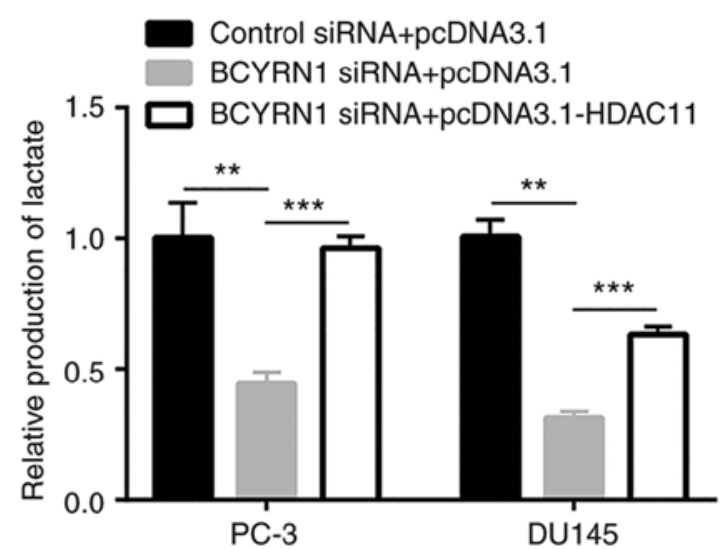

E PC-3
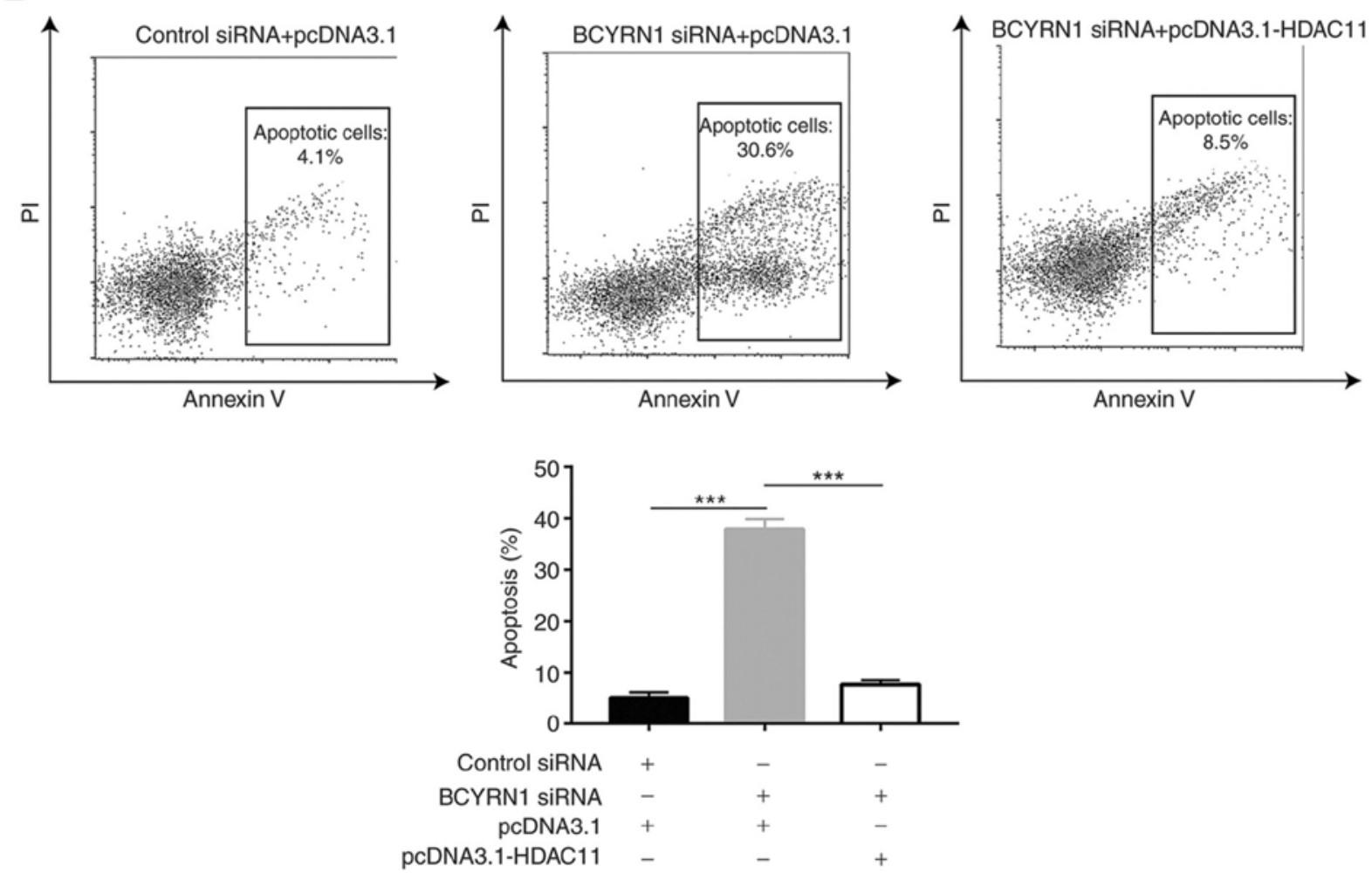

Figure 6. BCYRN1 promoted cell proliferation, glucose metabolism and cell survival via upregulation of HDAC11. (A) Overexpression of HDAC11 antagonized BCYRN1 silencing induced cell proliferation inhibition in PC-3 cells. (B) Overexpression of HDAC11 reversed BCYRN1 silencing induced cell proliferation inhibition in DU145 cells. (C) Overexpression of HDAC11 reversed BCYRN1 silencing induced glucose uptake inhibition in PC-3 and DU145 cells. (D) Overexpression of HDAC11 reversed BCYRN1 silencing induced lactate production inhibition in PC-3 and DU145 cells. (E) Overexpression of HDAC11 reversed BCYRN1 silencing induced cell apoptosis in PC-3 cells. 


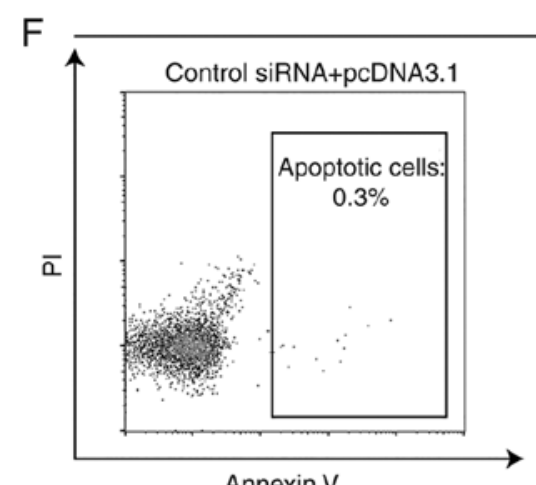

Annexin V
DU145
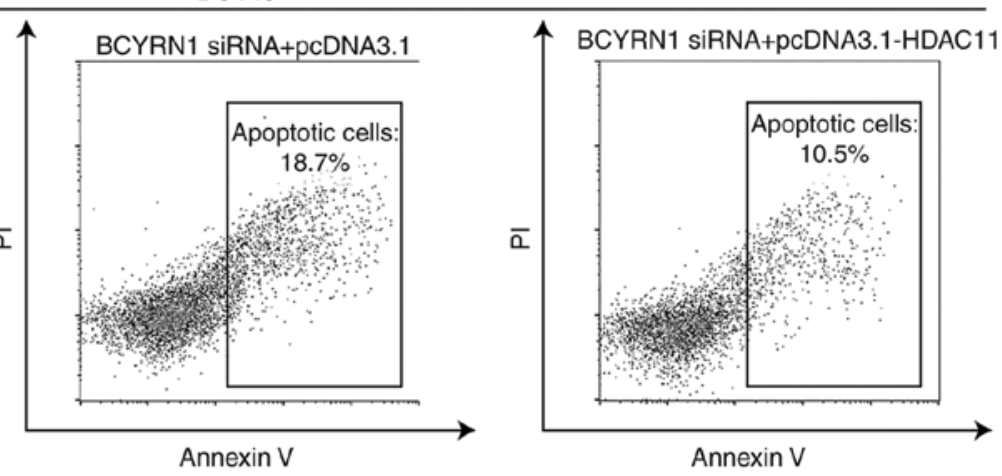

Annexin V

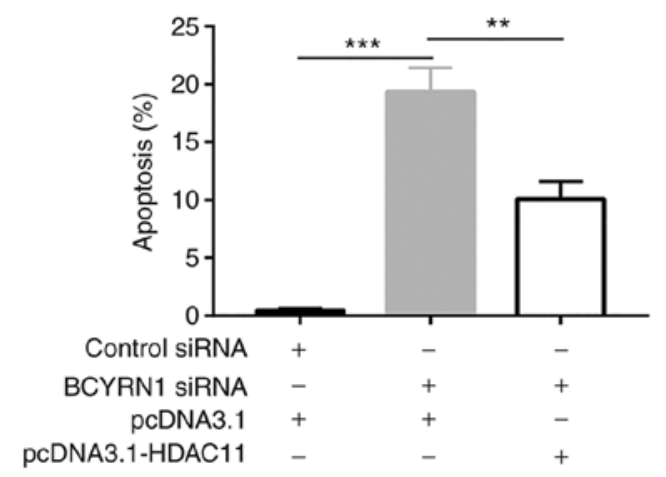

Figure 6. Continued. (F) Overexpression of HDAC11 reversed BCYRN1 silencing induced cell apoptosis in DU145 cells. ${ }^{* * *} \mathrm{P}<0.01{ }^{* * * *} \mathrm{P}<0.001$. BCYRN1, brain cytoplasmic RNA 1; HDAC11, histone deacetylase 11.

H4K16 in prostate cancer. To study the association between BCYRN1 and HDAC11 in prostate cancer, recombinant HDAC11 was transfected into PC-3 cells to upregulate HDAC11 expression (Fig. 5A). It was observed that HDAC11 promoted the progression of prostate cancer mainly via its deacetylation activity. Overexpression of HDAC11 also increased HDAC activity, while silencing of BCYRN1 reduced HDAC activity in PC-3 and DU145 cells (Fig. 5B and C). Due to abnormal HDAC activity, H3K9 and H4K16 were hypoacetylated, and the transcription of tumor suppressor genes was repressed in cancer (26). It was demonstrated that the global acetylation levels of H3K9 were elevated following BCYRN1 silencing, and this effect was reversed after HDAC11 overexpression in PC-3 and DU145 cells (Fig. 5D). Moreover, the global acetylation levels of H4K16 were elevated following BCYRN1 silencing, and this effect was reversed after HDAC11 overexpression in PC-3 and DU145 cells (Fig. 5E). Furthermore, the mRNA expression levels of CDKN1A and OPTN, two tumor suppressor genes tightly controlled by histone acetylation in prostate cancer (26), were increased after BCYRN1 silencing and decreased after HDAC11 overexpression in PC-3 and DU145 cells (Fig. 5F and G). Therefore, these results indicate that BCYRN1 regulates global histone acetylation via HDAC11 in prostate cancer.

BCYRN1 promotes cell proliferation, glucose metabolism and cell survival via upregulation of HDAC11. Cell proliferation assay results revealed that overexpression of HDAC11 reversed si-BCYRN1-induced proliferation inhibition in PC-3 and DU145 cells (Fig. 6A and B). In addition, HDAC11 upregulation reversed BCYRN1 silencing-induced inhibition of glucose uptake and lactate production in PC-3 and DU145 cells (Fig. 6C and D). Thus, BCYRN1 may promote glucose metabolism via the regulation of HDAC11 expression. Moreover, HDAC11 overexpression further inhibited apoptosis induced by BCYRN1 silencing in PC-3 and DU145 cells (Fig. 6E and F). Collectively, the results mentioned above indicate that BCYRN1 promotes prostate cancer progression via upregulation of HDAC11.

BCYRN1 is positively associated with HDAC11 in prostate tumor and healthy prostate tissues. To study the association between BCYRN1 and HDAC11 in clinical samples, HDAC11 mRNA expression was detected in 72 pairs of prostate tumors and healthy prostate tissues. The RT-qPCR analysis revealed an increase in HDAC11 levels in prostate tumors (Fig. 7A). Moreover, Pearson's correlation analysis revealed a strong positive correlation between BCYRN1 and HDAC11 mRNA expression levels in prostate tumors and healthy prostate tissues (Fig. 7B). Furthermore, RT-qPCR also demonstrated that miR-939-3p was decreased in prostate tumors, and a negative correlation was observed between miR-939-3p and BCYRN1 levels (Fig. 7C and D).

\section{Discussion}

Aberrant expression of BCYRN1 has been reported in several types of cancer, such as breast, bladder and colon cancer (27). However, the role of BCYRN1 in prostate cancer has not fully elucidated. Using bioinformatics analysis of TCGA-PRAD dataset, BCYRN1 was found to be one of the most significantly upregulated lncRNAs in prostate cancer. 
A

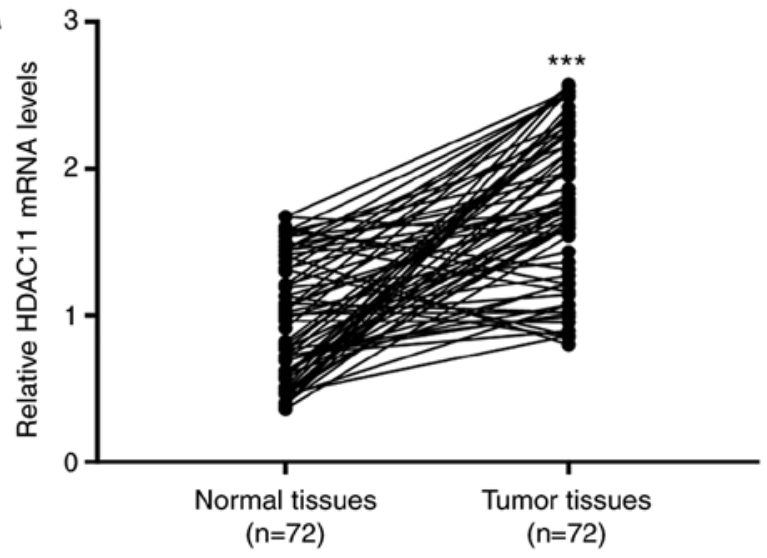

C

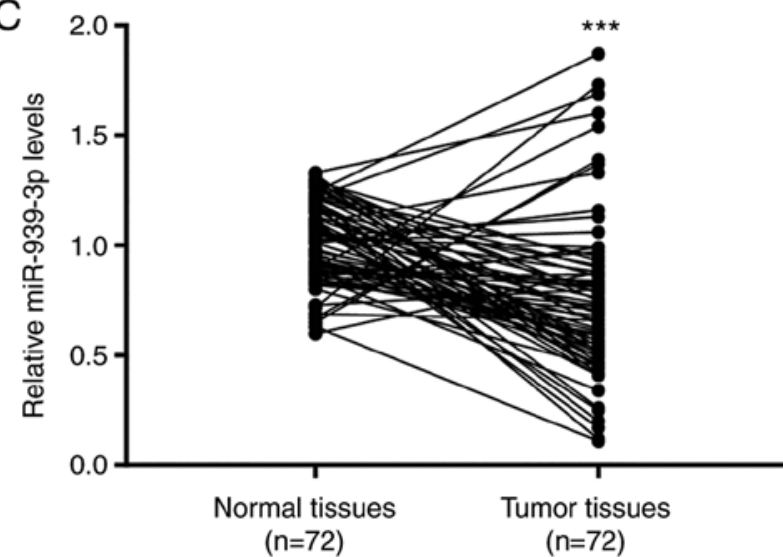

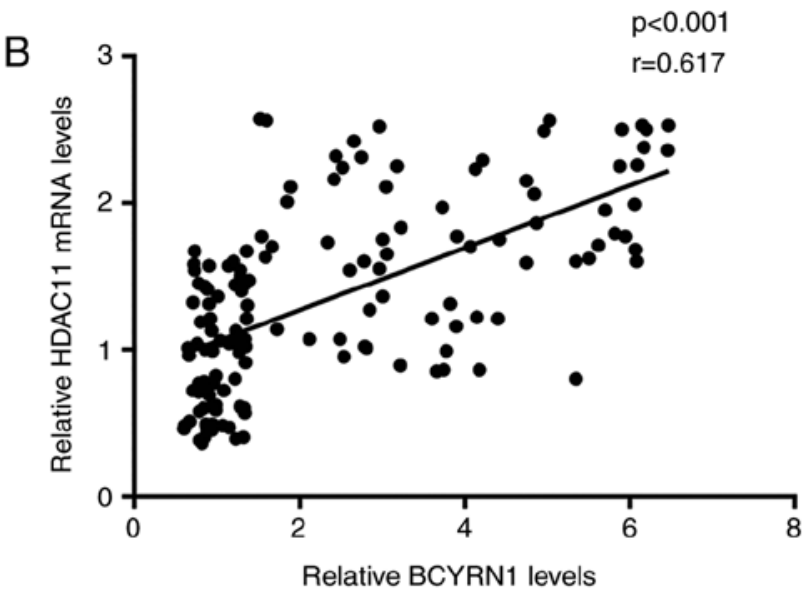

D

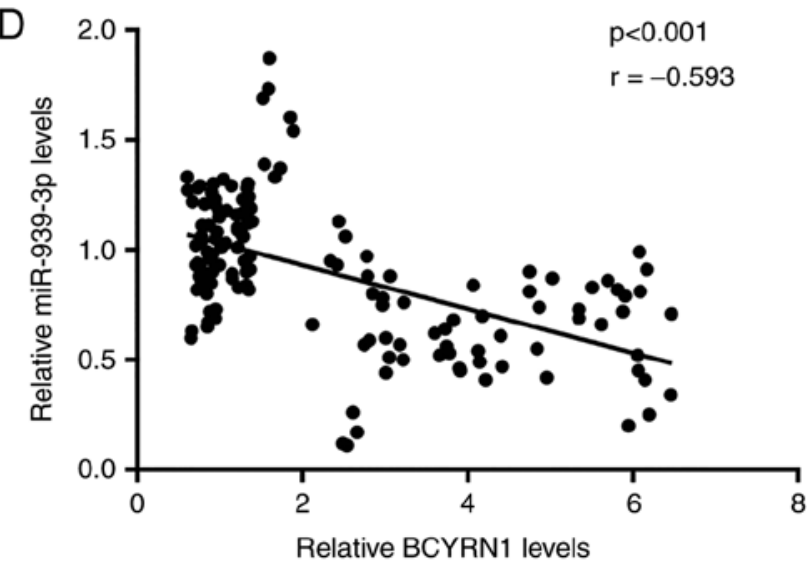

Figure 7. The expression of BCYRN1 was correlated with HDAC11 expression in clinical prostate samples. (A) RT-qPCR analysis revealed that HDAC11 mRNA expression was elevated in prostate tumors compared with that in matched normal tissues. (B) Pearson's correlation analysis indicated a positive correlation between BCYRN1 and HDAC11 mRNA expression in prostate tumors and normal prostate tissues. (C) RT-qPCR analysis revealed that miR-939-3p expression was decreased in prostate tumors compared with that in matched normal tissues. (D) Pearson's correlation analysis indicated a negative correlation between BCYRN1 and miR-939-3p expression in prostate tumors and normal prostate tissues. ${ }^{* * *} \mathrm{P}<0.001$. BCYRN1, brain cytoplasmic RNA 1; HDAC11, histone deacetylase 11; RT-qPCR, reverse transcription-quantitative PCR.

In addition, increased expression of BCYRN1 was detected in prostate cancers with a high Gleason score compared with those with a low Gleason score. Reprogrammed energy metabolism is a hallmark of cancer (28). Furthermore, abnormal glucose metabolism facilitates prostate cancer cell proliferation and survival (29). It was previously demonstrated that lncRNAs play pivotal roles in mediating glucose metabolism of cancer cells (30). The lncRNA urothelial cancer associated 1 promotes glucose metabolism via the mTOR-STAT3/miR-143-HK2 cascade in bladder cancer (31). In the present study, it was observed that BCYRN1 promoted glycolysis in prostate cancer cells. GLUT1, a crucial mediator of glucose metabolism in prostate cancer, was downregulated upon BCYRN1 silencing. Furthermore, BCYRN1 accelerated proliferation and facilitated survival of prostate cancer cells. It is possible that BCYRN1 sustains glycolysis to promote prostate cancer cell proliferation and survival. Collectively, the results of the present study suggested an oncogenic potential of BCYRN1 in prostate cancer. The biological function of BCYRN1 was investigated using a single siRNA-mediated gene knockdown. The role of BCYRN1 will be further explored by knockdown of BCYRN1 with two independent siRNAs in the future.
HDAC11 is a newly identified oncogene in several types of cancer (32). It was previously reported that HDAC11 is a crucial metabolic regulator (33). Furthermore, depletion of HDAC11 inhibits metabolism and promotes apoptosis in a variety of cancer cells, including prostate cancer cells (25). Moreover, the expression of HDAC11 is regulated by miRNAs (34). According to a meta-analysis based on 6 datasets, miR-939-3p is one of 22 commonly downregulated miRNAs in recurrent compared with non-recurrent prostate cancer samples (28). It has been demonstrated that lncRNA-HEIH promotes colorectal cancer progression via sponging miR-939 and upregulation of Bcl-xL (35). In ovarian cancer, however, miR-939 promoted cancer cell proliferation via APC2 targeting and activation of Wnt signaling (36). The results of the present study indicated that HDAC11 is a target gene of miR-939-3p in prostate cancer. Moreover, BCYRN1 sponged miR-939-3p to elevate HDAC11 levels in prostate cancer cells. As a deacetylase, HDAC11 controls gene expression via deacetylation of histone $\mathrm{H} 3$ and $\mathrm{H} 4$ (37). The results of the present study suggested that BCYRN1 deacetylated H3K9 and $\mathrm{H} 4 \mathrm{~K} 16$ via regulation of HDAC11 in prostate cancer cells. It has been demonstrated that acetylation of H3K9 and H4K16 regulates the transcription of tumor suppressors and oncogenes 
in cancer (26). Furthermore, hypoacetylation of H4K16 decreases the expression levels of the tumor suppressor genes CDKN1A and OPTN in prostate cancer (26). The expression of GLUT1 was also regulated by histone modification in prostate cancer. The RT-qPCR results demonstrated that BCYRN1 silencing decreased CDKN1A and OPTN mRNA expression levels, and these effects were reversed following HDAC11 overexpression in prostate cancer cells. Thus, BCYRN1 may inhibit tumor suppressor expression via histone modification. It was observed that HDAC11 overexpression reversed si-BCYRN1-induced inhibition of glucose metabolism, cell proliferation and apoptosis in prostate cancer cells. Therefore, it appears that the BCYRN1/miR-939-3p/HDAC11 axis may be involved in the regulation of key gene expression levels in prostate cancer cells, via controlling global histone acetylation. However, the biological role of BCYRN1 in the initiation, metastasis and development of prostate cancer in vivo was not investigated in the present study. BCYRN1 stable knockout prostate cancer cells will be established in the future to further study the function of BCYRN1 in vivo.

In conclusion, the results of the present study suggested that BCYRN1 is upregulated in prostate cancer, and that it promotes proliferation, glucose metabolism and survival of prostate cancer cells via sponging miR-939-3p and increasing the levels of HDAC11. Therefore, the present study may provide novel evidence that BCYRN1 may be of value as a biomarker and therapeutic target in prostate cancer.

\section{Acknowledgements}

Not applicable.

\section{Funding}

No funding was received.

\section{Availability of data and materials}

All the datasets generated and/or analyzed during the present study are available from the corresponding author on reasonable request.

\section{Authors' contributions}

WH, FQ and KW participated in the experimental design and data acquisition; FQ contributed to the collection of prostate specimens and clinical data; KW supervised the whole study and wrote the manuscript. All the authors have read and approved the final version of the manuscript for publication.

\section{Ethics approval and consent to participate}

All procedures performed in the present study involving human participants were approved by the Institutional Ethics Review Board of China-Japan Union Hospital.

\section{Patient consent for publication}

Written informed consent for the publication of all data was provided by all patients prior to surgery.

\section{Competing interests}

The authors declare that they have no competing interests.

\section{References}

1. Bray F, Ferlay J, Soerjomataram I, Siegel RL, Torre LA and Jemal A: Global cancer statistics 2018: GLOBOCAN estimates of incidence and mortality worldwide for 36 cancers in 185 countries. CA Cancer J Clin 68: 394-424, 2018.

2. Kaarbø M, Mikkelsen OL, Malerød L, Qu S, Lobert VH, Akgul G, Halvorsen T, Maelandsmo GM and Saatcioglu F: PI3K-AKT-mTOR pathway is dominant over androgen receptor signaling in prostate cancer cells. Cell Oncol 32: 11-27, 2010.

3. Bhardwaj S and Varma S: Rare incidence of tumor lysis syndrome in metastatic prostate cancer following treatment with docetaxel. J Oncol Pharm Pract 24: 153-155, 2018.

4. Cai Z, Wu Y, Li Y, Ren J and Wang L: BCAR4 activates GLI2 signaling in prostate cancer to contribute to castration resistance. Aging (Albany NY) 10: 3702-3712, 2018.

5. Wang XZ, Beebe JR, Pwiti L, Bielawska A and Smyth MJ: Aberrant sphingolipid signaling is involved in the resistance of prostate cancer cell lines to chemotherapy. Cancer Res 59: 5842-5848, 1999.

6. Chen X and Yan GY: Novel human lncRNA-disease association inference based on lncRNA expression profiles. Bioinformatics 29: 2617-2624, 2013.

7. McHugh CA, Chen CK, Chow A, Surka CF, Tran C, McDonel P, Pandya-Jones A, Blanco M, Burghard C, Moradian A, et al: The Xist lncRNA interacts directly with SHARP to silence transcription through HDAC3. Nature 521: 232-236, 2015.

8. Yang Q, Wan Q, Zhang L, Li Y, Zhang P, Li D, Feng C, Yi F, Zhang L, Ding X, et al: Analysis of LncRNA expression in cell differentiation. RNA Biol 15: 413-422, 2018.

9. Qiu M, Xu Y, Wang J, Zhang E, Sun M, Zheng Y, Li M, Xia W, Feng D, Yin R and Xu L: A novel lncRNA, LUADT1, promotes lung adenocarcinoma proliferation via the epigenetic suppression of p27. Cell Death Dis 6: e1858, 2015.

10. Su M, Xiao Y, Tang J, Wu J, Ma J, Tian B, Zhou Y, Wang H, Yang D, Liao QJ and Wang W: Role of IncRNA and EZH2 interaction/regulatory network in lung cancer. J Cancer 9: 4156-4165, 2018.

11. Zan XY and Li L: Construction of IncRNA-mediated ceRNA network to reveal clinically relevant lncRNA biomarkers in glioblastomas. Oncol Lett 17: 4369-4374, 2019.

12. Du Y, Weng XD, Wang L, Liu XH, Zhu HC, Guo J, Ning JZ and Xiao CC: LncRNA XIST acts as a tumor suppressor in prostate cancer through sponging miR-23a to modulate RKIP expression. Oncotarget 8: 94358-94370, 2017.

13. Lingadahalli S, Jadhao S, Sung YY, Chen M, Hu L, Chen X and Cheung E: Novel lncRNA LINC00844 regulates prostate cancer cell migration and invasion through AR signaling. Mol Cancer Res 15: 1865-1878, 2018.

14. Gu P, Chen X, Xie R, Xie W, Huang L, Dong W, Han J, Liu X, Shen J, Huang J and Lin T: A novel AR translational regulator lncRNA LBCS inhibits castration resistance of prostate cancer. Mol Cancer 18: 109, 2019.

15. Shin H, Kim Y, Kim M and Lee Y: BC200 RNA: An emerging therapeutic target and diagnostic marker for human cancer. Mol Cells 41: 993-999, 2018.

16. Wu K, Xu K, Liu K, Huang J, Chen J, Zhang J and Zhang N: Long noncoding RNA BC200 regulates cell growth and invasion in colon cancer. Int J Biochem Cell Biol 99: 219-225, 2018.

17. Zhao RH, Zhu CH, Li XK, Cao W, Zong H, Cao XG and Hu HY: BC200 LncRNA a potential predictive marker of poor prognosis in esophageal squamous cell carcinoma patients. Onco Targets Ther 9: 2221-2226, 2016.

18. Iacoangeli A, Adzovic L, Chen EQ, Latif Cattie R, Soff GA and Tiedge H: Regulatory BC200 RNA in peripheral blood of patients with invasive breast cancer. J Investig Med 66: 1055-1063, 2018.

19. Thole TM,Lodrini M, Fabian J, Wuenschel J, Pfeil S, Hielscher T, Kopp-Schneider A, Heinicke U, Fulda S, Witt Om, et al: Neuroblastoma cells depend on HDAC11 for mitotic cell cycle progression and survival. Cell Death Dis 8: e2635, 2017.

20. Byun SK, An TH, Son MJ, Lee DS, Kang HS, Lee EW, Han BS, Kim WK, Bae KH, Oh KJ and Lee SC: HDAC11 inhibits myoblast differentiation through repression of MyoD-dependent transcription. Mol Cells 40: 667-676, 2017. 
21. Deubzer HE, Schier MC, Oehme I, Lodrini M, Haendler B Sommer A and Witt O: HDAC11 is a novel drug target in carcinomas. Int J Cancer 132: 2200-2208, 2013.

22. Livak KJ and Schmittgen TD. Analysis of relative gene expression data using real-time quantitative PCR and the 2(-Delta Delta C(T)) method. Methods 25: 402-408, 2001.

23. Vaz CV, Alves MG, Marques R, Moreira PI, Oliveira PF, Maia CJ and Socorro S: Androgen-responsive and nonresponsive prostate cancer cells present a distinct glycolytic metabolism profile. Int J Biochem Cell Biol 44: 2077-2084, 2012.

24. Pashaei E, Pashaei E, Ahmady M, Ozen M and Aydin N: Meta-analysis of miRNA expression profiles for prostate cancer recurrence following radical prostatectomy. PLoS One 12: e0179543, 2017.

25. Guo LL, Song CH, Wang P, Dai LP, Zhang JY and Wang KJ Competing endogenous RNA networks and gastric cancer. World J Gastroenterol 21: 11680-11687, 2015.

26. Katoh H, Qin ZS, Liu R, Wang L, Li W, Li X, Wu L, Du Z, Lyons R, Liu CG, et al: FOXP3 orchestrates H4K16 acetylation and H3K4 trimethylation for activation of multiple genes by recruiting MOF and causing displacement of PLU-1. Mol Cell 44: 770-784, 2011.

27. Samson J, Cronin S and Dean K: BC200 (BCYRN1)-The shortest, long, non-coding RNA associated with cancer. Noncoding RNA Res 3: 131-143, 2018.

28. Hanahan D and Weinberg RA: Hallmarks of cancer: The next generation. Cell 144: 646-674, 2011.

29. Yang B, Zhang L, Cao Y, Chen S, Cao J, Wu D, Chen J, Xiong H, Pan Z, Qiu F, et al: Overexpression of lncRNA IGFBP4-1 reprograms energy metabolism to promote lung cancer progression. Mol Cancer 16: 154, 2017
30. Chen $\mathrm{S}, \mathrm{Xu} \mathrm{X}, \mathrm{Lu} \mathrm{S}$ and $\mathrm{Hu} \mathrm{B}$ : Long non-coding RNA HAND2-AS1 targets glucose metabolism and inhibits cancer cell proliferation in osteosarcoma. Oncol Lett 18: 1323-1329, 2019.

31. Li Z, Li X, Wu S, Xue M and Chen W: Long non-coding RNA UCA1 promotes glycolysis by upregulating hexokinase 2 through the mTOR-STAT3/microRNA143 pathway. Cancer Sci 105: 951-955, 2014.

32. Leslie PL, Chao YL, Tsai YH, Ghosh SK, Porrello A, Van Swearingen AED, Harrison EB, Cooley BC, Parker JS, Carey LA and Pecot CV: Histone deacetylase 11 inhibition promotes breast cancer metastasis from lymph nodes. Nat Commun 10: 4192, 2019.

33. Bhaskara S: Histone deacetylase 11 as a key regulator of metabolism and obesity. EBioMedicine 35: 27-28, 2018.

34. Lin L, Hou J, Ma F, Wang P, Liu X, Li N, Wang J, Wang Q and Cao X: Type I IFN inhibits innate IL-10 production in macrophages through histone deacetylase 11 by downregulating microRNA-145. J Immunol 191: 3896-3904, 2013.

35. Cui C, Zhai D, Cai L, Duan Q, Xie L and Yu J: Long noncoding RNA HEIH promotes colorectal cancer tumorigenesis via counteracting miR-939 mediated transcriptional repression of Bcl-xL. Cancer Res Treat 50: 992-1008, 2018.

36. Ying X, Liya Q, Feng Z, Yin W and Ji-hong L: MiR-939 promotes the proliferation of human ovarian cancer cells by repressing APC2 expression. Biomed Pharmacother 71: 64-69, 2015.

37. Liu H, Hu Q, D'Ercole AJ and Ye P: Histone deacetylase 11 regulates oligodendrocyte-specific gene expression and cell development in OL-1 oligodendroglia cells. Glia 57: 1-12, 2009. 